\title{
Weed diversity in tomato crops in the mountainous region of South Kivu, DR Congo
}

\author{
Victoire M. Aganze ${ }^{(1,2)}$, Marcellin C. Cokola ${ }^{(3)}$, Justin M. Salimbasi ${ }^{(2)}$, Arnaud Monty ${ }^{(4)}$ \\ (1) Université Catholique de Bukavu (UCB). Faculty of Agronomy (FA). Department of Crop Sciences. Bukavu, South Kivu \\ (DR Congo). E-mail: victoiremunene@gmail.com \\ (2) Institut supérieur d'Études Agronomiques et Vétérinaires. Section of Crop Production. Kabare, South Kivu (DR Congo). \\ (3) Université Évangélique en Afrique. Faculty of Agronomy (FA). Department of Crop Sciences. Bukavu, South Kivu (DR \\ Congo). \\ (4) Université de Liège - Gembloux Agro-Bio Tech. TERRA Research Center. Gembloux (Belgium).
}

Received 14 November 2019, accepted 23 September 2020, available online 28 September 2020.

This article is distributed under the terms and conditions of the CC-BY License (http://creativecommons.org/licenses/by/4.0)

Description of the subject. Despite the importance of tomato in Central Africa, little is known about the weed flora occurring in tomato crops in this part of the world.

Objectives. We aimed to provide a comprehensive description of the weed flora found in tomato crops in the mountainous region of South Kivu, DR Congo.

Method. Floristic inventories were made in four cultivation zones of the Kabare territory, South Kivu.

Results. In total, 79 species (belonging to 64 genera and 31 families) were recorded. The most species-rich families were Asteraceae and Poaceae. Therophytes and geophytes were the dominant life forms. Weed diversity was not significantly different between sites but species composition varied considerably, likely in relation to soil type. Based on the frequency and the abundance, the most problematic weeds were Commelina benghalensis, Galinsoga quadriradiata, Commelina diffusa, Galinsoga parviflora, Bidens pilosa, Oxalis debilis and Digitaria velutina.

Conclusions. The weed flora in tomato fields of the mountainous regions of DR Congo is diversified, but only a few cosmopolitan species are problematic.

Keywords. Vegetable crops, agriculture, cultivation, noxious plants, Central Africa.

\section{Flore adventice de la tomate dans le Sud-Kivu montagneux, RD Congo}

Description du sujet. En dépit de l'importance de la culture de tomates en Afrique centrale, peu d'études sur la flore adventice de cette culture y ont été réalisées.

Objectifs. Cette étude visait à caractériser la flore adventice des cultures de tomates dans les régions montagneuses du SudKivu, RD Congo.

Méthode. Des inventaires floristiques ont été réalisés dans quatre zones de culture du territoire de Kabare, Sud-Kivu.

Résultats. Au total, 79 espèces, réparties en 64 genres et 31 familles, ont été inventoriées. Les Asteraceae et les Poaceae constituent les familles les plus diversifiées. La flore est dominée par les thérophytes et les géophytes. La diversité spécifique était comparable entre les zones de culture, mais la composition en espèces y était variable. Sept espèces d'importance agronomique ont été identifiées : Commelina benghalensis, Galinsoga quadriradiata, Commelina diffusa, Galinsoga parviflora, Bidens pilosa, Oxalis debilis and Digitaria velutina.

Conclusions. La flore adventice de la tomate dans les régions montagneuses du Sud-Kivu est diversifiée. Néanmoins, les quelques espèces problématiques sont des espèces cosmopolites.

Mots-clés. Plante légumière, agriculture, pratique culturale, plante nuisible, Afrique centrale.

\section{INTRODUCTION}

Tomato (Solanum lycopersicum L.) is a widely grown vegetable in Central Africa, where it provides important income to smallholder farmers (Chausse et al., 2012).
Tomato yields in DR Congo are low (ca 7.5 ton $\cdot \mathrm{ha}^{-1}$ ) as compared to world leading countries such as China or India (FAOSTAT, 2019). This is attributable to several factors, notably weed competition and pests damages. Through competition for water, light, and 
nutrients, weeds are a major yield-limiting constraint (Nichols et al., 2015). In contrast to other pests, they are ubiquitous in agricultural systems. Adigun (2005) attributed a 40 to $60 \%$ decrease in tomato fruit yield to weed pressure in Nigeria. Likewise, Amare et al. (2015) recorded ca 90\% yield loss caused by early weed competition in Ethiopia. In Central Africa, where hand-weeding is the dominant weed management method, smallholder farmers spend up to $50 \%$ of agricultural activities to control weeds (Ellis-Jones et al., 1993). Several studies (e.g. Le Bourgeois, 1993; Noba et al., 2004) emphasized the need for a better knowledge of weed infestation as a key step for setting up an efficient management strategy. However, no study to date has been carried out on weed pressure in vegetable crops, especially tomato, in the mountainous regions of Democratic Republic of the Congo. The main goal of this research was to describe the weed community occurring in tomato crop and to identify the most problematic, i.e. the most frequent and abundant ones. Our results can be used to provide guidance for weed management strategy in this part of the world.

\section{MATERIALS AND METHODS}

\subsection{Study sites}

This study was carried from March to June 2019 in four cultivation zones (Figure 1) that are representative of the soil variation in South Kivu. According to Wils et al. (1986), the mountainous region of Kivu can be divided into two soil types: volcanic soils, usually highly productive; and ferralitic sedimentary soils, with low to medium fertility. The cultivation zones of Luhihi (altitude ca 1,695 m) and Bugorhe (altitude ca $1,712 \mathrm{~m})$ are implemented on volcanic soils, whereas Mudaka (altitude ca 1,588 m) and Bushwira (altitude ca 1,646 m) are implemented on ferralitic soils. The distances between cultivation zones varied between $3.9 \mathrm{~km}$ (Bushwira-Mudaka) and $18.7 \mathrm{~km}$ (Bushwira-Luhihi). The study area is characterized by a tropical mountain climate with a long rainy season (from September to May) and a short dry season (June to August). Mean daily temperature ranges from 17 to $19.5^{\circ} \mathrm{C}$. Annual rainfall is ca $1,400 \mathrm{~mm}$.

\subsection{Field records}

A preliminary study was achieved to determine the minimum quadrat size according to Gillet (2000), and $4 \mathrm{~m}^{2}$ was the minimum area retained. On-field floristic inventories were then conducted in 10 tomato fields (all planted in March 2019) in each four cultivation zones, one month after tomato plantation/sowing and before the fields were weeded. In each field, three $2 \mathrm{~m} \times 2 \mathrm{~m}$ quadrats were placed randomly and all plant species were recorded. The three quadrats per field were pooled prior to analyses. Cover was assessed using Braun-Blanquet (1952) scale, as a proxy for abundance. Complementary samplings were made 2 and 3 months after tomato planting, in the same quadrats, to take into account new species. Tomato was implemented after another culture in 30 out of the 40 fields. The most common previous crops were beans, maize and onions. Continuous tomato cultivation was more common

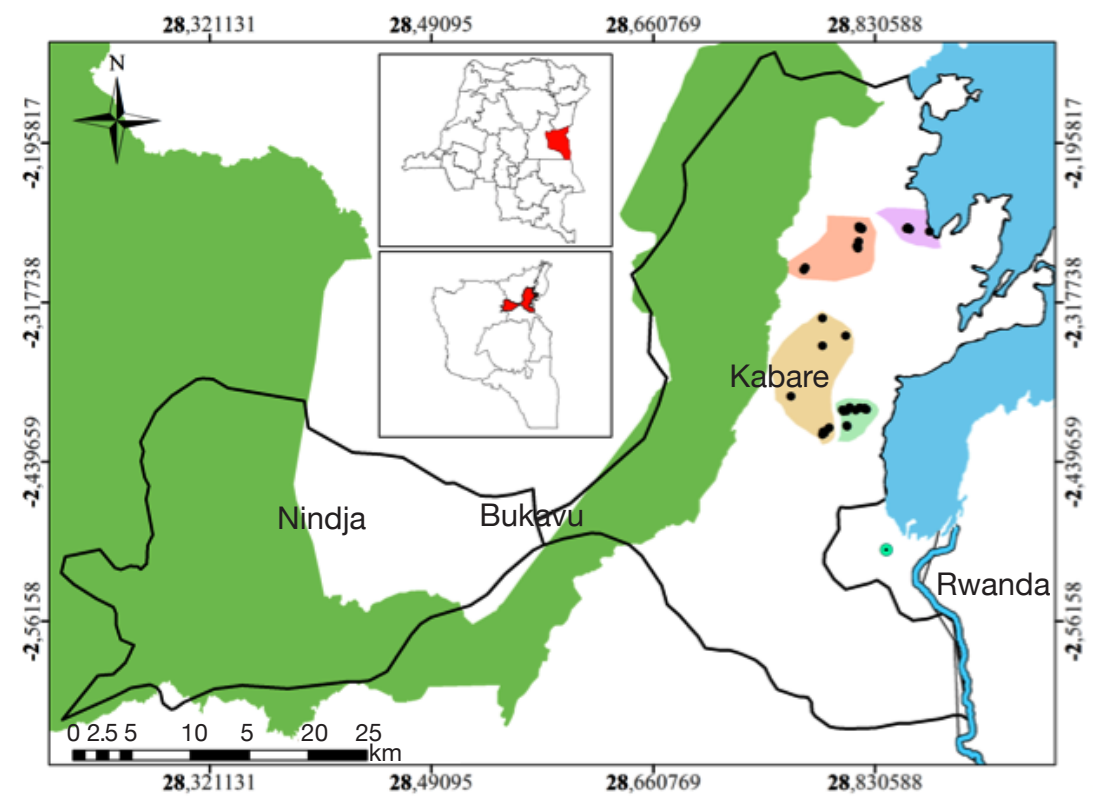

\section{Legend}

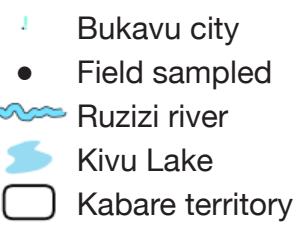

Mudaka

Bushwira

Bugorhe

Luhihi

PNKB, Kahuzi-Biega National Park

Figure 1. Presentation of Kabare territory - Carte du territoire de Kabare. 
in Mudaka (5 fields) compared to Bushwira (1 field), Luhihi (2 fields) and Bugorhe (2 fields).

\subsection{Data analysis}

The "infestation diagram" approach (Le Bourgeois, 1993; Lebreton \& Le Bourgeois, 2005) was used to identify the most problematic weed species, i.e. the most frequent and abundant ones: general majors species are very ubiquitous (RF $>50 \%$ ) and abundant (cover $>14 \%)$; general species are also frequent $(\mathrm{RF}>$ $50 \%$ ) but less abundant (cover $<14 \%$ ). Other species are considered less problematic. Before calculation, Braun-Blanquet values were transformed in $\%$ using the abundance (AD) index table of Gillet (2000): $0.03 \%$ (r); $0.03 \%(+) ; 3 \%(1) ; 14 \%(2) ; 32 \%(3) ; 57 \%(4)$ and $90 \%$ (5). Then, the abundance average (AD av) was calculated using the following formula:

$$
\mathrm{AD} \text { av }=\sum \mathrm{AD} / \mathrm{AF}
$$

with $\mathrm{AF}=$ absolute frequency; $\sum \mathrm{AD}=$ sum of abundance index of a species in all quadrats. We estimated $\alpha$-diversity through species richness and performed a one-way ANOVA to evaluate the effect of cultivation zones. A two-dimensional non-metric multi-dimensional scaling (NMDS) ordination was run on the Bray-Curtis similarity matrix (based on species abundance) with all plots, using the vegan package (Oksanen et al., 2013). Analyses were performed using $\mathrm{R}$ statistical software (R Development Core Team 2010).

\section{RESULTS}

A total of 79 species, belonging to 64 genera and 31 families, were recorded (Table 1). All belong to angiosperms (of which $69.2 \%$ eudicots and 30.8\% monocots). Seven families represented more than half of the species: Asteraceae (14 species); Poaceae (9 species); Amaranthaceae (5 species); Fabaceae (5 species); Solanaceae (5 species); Cyperaceae (4 species) and Malvaceae (4 species). The analysis of life forms revealed the predominance of therophytes (47.4\% of species richness) and geophytes (18.0\%). Species richness varied between cultivation zones $(\mathrm{df}=3 ; \mathrm{F}=$ $3.2, p=0.03$ ): it was significantly higher in Mudaka (17.6 \pm 4.3 species) compared to Bushwira (12.9 \pm 3.4 species), but not significantly different to Bugorhe (16 \pm 2.2 species) and Luhihi (16.2 \pm 3.9 species).

General species were Commelina benghalensis L., Galinsoga quadriradiata Ruiz \& Pav., Commelina diffusa Burm.f., Galinsoga parviflora Cav., Bidens pilosa L., Digitaria velutina (Forssk.) P.Beauv. and Oxalis debilis Kunth (Figure 2). Amaranthus hybridus
L. was also frequent $(>50 \%)$. All other species had much lower infestation levels. Among them, several are cultivated (e.g. Colocasia esculenta (L.) Schott, Zea mays L., Manihot esculenta Crantz) or used in traditional medicine (Erucastrum arabicum Fisch. \& C.A.Mey., Oxalis corniculata L., Ricinus communis L., Achyranthes aspera L., Bothriocline longipes [Oliv. \& Hiern] N.E.Br., Cleome gynandra L., Crassocephalum montuosum [S.Moore] Milne-Redh.).

The NMDS plot (Figure 3) showed an overall separation between the weed flora of the cultivation zones on ferralitic sedimentary soils (Mudaka and Bushwira) vs volcanic soils (Luhihi and Bugorhe).

\section{DISCUSSION}

The number of species identified during this study (79 species) may seem low as compared to the flora of DR Congo, but it is much higher than those reported by Mushagalusa et al. (2014) in corn crops (46 species) and Miderho et al. (2018) in coffee trees (18 species), in mountainous regions of DR Congo. The most species-rich families recorded (Asteraceae and Poaceae) belong to the most ubiquitous weed families in agrosystems (Zimdahl, 2007; Jauzein, 2011). In regularly disturbed ecosystems such as cultivated fields, therophytes usually dominate (Noba et al., 2004; Reboud et al., 2009), which was clearly the case in our study. However, a significant proportion of species were geophytes, essentially due to the dominance of cultivated plants (e.g. Allium cepa L., Musa spp., C. esculenta, Xanthosoma sagittifolium (L.) Schott, Ipomoea batatas (L.) Lam., Dioscorea alata L., Solanum tuberosum L.).

The most problematic species identified, except G. parviflora, D. velutina and C. benghalensis, have already been reported by Mushagalusa et al. (2014) and/or Miderho et al. (2018). Most of them are cosmopolitan, listed worldwide as problematic weeds (Jauzein, 2011; Le Bourgeois et al., 2015). The species A. hybridus can be considered as less noxious, as it is a vegetable much appreciated by famers and purposely left in fields. Thus, problematic species can be reduced to seven (G. quadriradiata, C. benghalensis, C.diffusa, G. parviflora, B.pilosa, O.debilis and $D$. velutina). They show high growth capacity and high seed production (Le Bourgeois et al., 2015). In addition, $C$. diffusa, $C$. bengalensis and $O$. debilis can spread by vegetative reproduction (Le Bourgeois et al., 2015). Locally, Rumex abyssinicus Jacq. and Cynodon dactylon (L.) Pers. are real weed problems.

Soil characteristic can be considered as a factor that likely affects weed composition in the studied region. Fried et al. (2008) and Zidane et al. (2010) reported similar observation in other parts of the world. 
Table 1. Weed-species identified in tomato fields during the study - Liste des espèces identifiées dans les champs de tomate au cours de l'étude.

\begin{tabular}{|c|c|c|c|c|c|}
\hline Family & Species & EPPO code & L-F & AD av & $\mathbf{R F}$ \\
\hline Acanthaceae & Hygrophila auriculata (Schumach.) Heine & HYGAU & $\mathrm{T}$ & 0.165 & 5 \\
\hline \multirow[t]{5}{*}{ Amaranthaceae } & Achyranthes aspera L. & ACYAS & $\mathrm{T}$ & 0.3 & 5 \\
\hline & Amaranthus cruentus L. & AMACR & $\mathrm{T}$ & 4.42 & 40 \\
\hline & Amaranthus hybridus L. & AMACH & $\mathrm{T}$ & 2.81 & 82.5 \\
\hline & Amaranthus spinosus L. & AMASP & $\mathrm{T}$ & 0.84 & 10 \\
\hline & Amaranthus viridis $\mathrm{L}$. & AMAVI & $\mathrm{T}$ & 3.24 & 32.5 \\
\hline Amaryllidaceae & Allium cepa $\mathrm{L}$. & ALLCE & G & 0.21 & 5 \\
\hline Anacardiaceae & Mangifera indica L. & MNGIN & $\mathrm{P}$ & 0.03 & 2.5 \\
\hline \multirow[t]{2}{*}{ Araceae } & Colocasia esculenta (L.) Schott & CXSES & $\mathrm{G}$ & 0.45 & 72.5 \\
\hline & Xanthosoma sagittifolium (L.) Schott & XATSA & $\mathrm{G}$ & 0.17 & 25 \\
\hline Asparagaceae & Dracaena afromontana Mildbr. & DRNAF & $\mathrm{P}$ & 0.03 & 2.5 \\
\hline \multirow[t]{14}{*}{ Asteraceae } & Acanthospermum hispidum DC. & ACNHI & $\mathrm{T}$ & 1.08 & 15 \\
\hline & Ageratum conyzoides L. & AGECO & $\mathrm{T}$ & 0.35 & 45 \\
\hline & Bidens pilosa $\mathrm{L}$. & BIDPI & $\mathrm{T}$ & 3.32 & 77.5 \\
\hline & Bothriocline longipes (Oliv. \& Hiern) N.E.Br. & BTCLO & $\mathrm{P}$ & 0.17 & 7.5 \\
\hline & Erigeron sumatrensis Retz. & ERISU & $\mathrm{T}$ & 0.17 & 5 \\
\hline & Crassocephalum montuosum (S.Moore) Milne-Redh. & CRAMO & $\mathrm{T}$ & 0.17 & 5 \\
\hline & Crassocephalum vitellinum (Benth.) S.Moore & CRAVI & $\mathrm{T}$ & 0.3 & 2.5 \\
\hline & Dichrocephala integrifolia (L.f.) Kuntze & DILIN & $\mathrm{T}$ & 0.3 & 10 \\
\hline & Emilia caespitosa Oliv. & EMICA & $\mathrm{T}$ & 0.3 & 5 \\
\hline & Galinsoga quadriradiata Ruiz \& Pav. & GASCI & $\mathrm{T}$ & 8.48 & 80 \\
\hline & Galinsoga parviflora Cav. & GASPA & $\mathrm{T}$ & 5.47 & 95 \\
\hline & Acmella caulirhiza Delile & SPLMR & $\mathrm{T}$ & 1.65 & 5 \\
\hline & Tagetes minuta $\mathrm{L}$. & TAGMI & $\mathrm{T}$ & 2.64 & 12.5 \\
\hline & Tithonia diversifolia (Hemsl.) A.Gray & TITDI & $\mathrm{Ch}$ & 0.3 & 2.5 \\
\hline Boraginaceae & Symphytum officinale L. & SYMOF & Hem & 0.03 & 2.5 \\
\hline \multirow[t]{3}{*}{ Brassicaceae } & Brassica oleracea L. & BRSOX & Hem & 0.82 & 5 \\
\hline & Cardamine africana $\mathrm{L}$. & CARAF & $\mathrm{T}$ & 0.98 & 5 \\
\hline & Erucastrum arabicum Fisch. \&C.A.Mey. & ERWAR & $\mathrm{T}$ & 1.14 & 40 \\
\hline Cannaceae & Canna indica $\mathrm{L}$. & CNNIN & G & 0.91 & 10 \\
\hline Cleomaceae & Cleome gynandra $\mathrm{L}$. & GYAGY & $\mathrm{T}$ & 0.12 & 7.5 \\
\hline \multirow[t]{3}{*}{ Commelinaceae } & Commelina africana $\mathrm{L}$. & COMAF & $\mathrm{Ch}$ & 3.73 & 5 \\
\hline & Commelina benghalensis L. & COMBE & $\mathrm{Ch}$ & 10.26 & 72.5 \\
\hline & Commelina diffusa Burm.f. & COMDI & $\mathrm{Ch}$ & 5.74 & 85 \\
\hline Convolvulaceae & Ipomoea batatas (L.) Lam. & IPOBA & $\mathrm{G}$ & 0.77 & 10 \\
\hline Cucurbitaceae & Cucurbita pepo L. & CUUPE & $\mathrm{T}$ & 0.12 & 10 \\
\hline \multirow[t]{4}{*}{ Cyperaceae } & Cyperus distans L.f. & CYPDT & G & 2.63 & 25 \\
\hline & Cyperus esculentus L. & CYPES & $\mathrm{G}$ & 1.41 & 35 \\
\hline & Cyperus latifolius Poir. & CYPLF & Hem & 1.10 & 15 \\
\hline & Cyperus spp. & CYPSP & & 2.76 & 45 \\
\hline
\end{tabular}


Table 1 (continued). Weed-species identified in tomato fields during the study - Liste des espèces identifiées dans les champs de tomate au cours de l'étude.

\begin{tabular}{|c|c|c|c|c|c|}
\hline Family & Species & EPPO code & L-F & AD av & $\mathbf{R F}$ \\
\hline Dioscoreaceae & Dioscorea alata $\mathrm{L}$. & DIUAL & $\mathrm{G}$ & 0.03 & 5 \\
\hline \multirow[t]{2}{*}{ Euphorbiaceae } & Manihot esculenta Crantz & MANES & $\mathrm{Ch}$ & 0.26 & 45 \\
\hline & Ricinus communis $\mathrm{L}$. & RIICO & $\mathrm{P}$ & 0.1 & 15 \\
\hline \multirow[t]{5}{*}{ Fabaceae } & Alysicarpus rugosus (Willd.) DC. & ALZRU & $\mathrm{T}$ & 0.72 & 30 \\
\hline & Arachis hypogaea $\mathrm{L}$. & ARHHY & $\mathrm{T}$ & 2.01 & 7.5 \\
\hline & Glycine $\max (\mathrm{L}$.$) Merr.$ & GLXMA & $\mathrm{T}$ & 0.03 & 5 \\
\hline & Indigofera arrecta A.Rich. & INDAR & $\mathrm{Ch}$ & 0.12 & 5 \\
\hline & Phaseolus vulgaris $\mathrm{L}$. & PHSVX & $\mathrm{T}$ & 0.12 & 25 \\
\hline Lamiaceae & Tetradenia riparia (Hochst.) Codd & TXDRI & $\mathrm{P}$ & 0.3 & 2.5 \\
\hline Lauraceae & Persea americana Mill. & PEBAM & $\mathrm{P}$ & 0.03 & 2.5 \\
\hline \multirow[t]{4}{*}{ Malvaceae } & Alcea rosea $\mathrm{L}$. & ALGRO & Hem & 3 & 2.5 \\
\hline & Hibiscus cannabinus L. & HIBCA & $\mathrm{T}$ & 1.51 & 22.5 \\
\hline & Sida acuta Burm.f. & SIDAC & $\mathrm{Ch}$ & 0.17 & 10 \\
\hline & Sida rhombifolia L. & SIDRH & $\mathrm{Ch}$ & 0.17 & 5 \\
\hline Moraceae & Ficus vallis-choudae Delile & FICVA & $\mathrm{P}$ & 0.03 & 2.5 \\
\hline \multirow[t]{2}{*}{ Musaceae } & Musa acuminata Colla & MUBAC & G & 0.03 & 5 \\
\hline & Musa paradisiaca $\mathrm{L}$. & MUSPA & G & 0.03 & 7.5 \\
\hline \multirow[t]{2}{*}{ Oxalidaceae } & Oxalis corniculata $\mathrm{L}$. & OXACO & $\mathrm{G}$ & 0.98 & 10 \\
\hline & Oxalis debilis Kunth & OXADE & $\mathrm{T}$ & 3.53 & 95 \\
\hline Phyllanthaceae & Phyllanthus niruri L. & PYLNI & $\mathrm{T}$ & 0.03 & 2.5 \\
\hline \multirow[t]{9}{*}{ Poaceae } & Cynodon dactylon (L.) Pers. & CYNDA & Hem & 7.15 & 5 \\
\hline & Digitaria abyssinica (A.Rich.) Stapf & DIGSC & G & 5.12 & 22.5 \\
\hline & Digitaria velutina (Forssk.) P.Beauv. & DIGVE & $\mathrm{T}$ & 1.3 & 85 \\
\hline & Eleusine indica (L.) Gaertn. & ELEIN & $\mathrm{T}$ & 0.45 & 17.5 \\
\hline & Eragrostis tenuifolia (A.Rich.) Hochst. ex Steud. & ERATE & Hem & 0.03 & 2.5 \\
\hline & Saccharum officinarum L. & SACOF & $\mathrm{Ch}$ & 2.5 & 10 \\
\hline & Setaria barbata (Lam.) Kunth & SETBA & $\mathrm{T}$ & 1.63 & 20 \\
\hline & Sorghum bicolor (L.) Moench & SORVU & $\mathrm{T}$ & 0.03 & 2.5 \\
\hline & Zea mays $\mathrm{L}$. & ZEAMX & $\mathrm{T}$ & 0.24 & 47.5 \\
\hline \multirow[t]{2}{*}{ Polygonaceae } & Persicaria senegalensis (Meisn.) Soják & POLSG & $\mathrm{Ch}$ & 0.3 & 5 \\
\hline & Rumex abyssinicus Jacq. & RUMAB & G & 8.23 & 5 \\
\hline Portulacaceae & Portulaca oleracea L. & POROL & $\mathrm{T}$ & 0.77 & 20 \\
\hline Rosaceae & Fragaria vesca $\mathrm{L}$. & FRAVE & $\mathrm{Ch}$ & 0.03 & 2.5 \\
\hline \multirow[t]{5}{*}{ Solanaceae } & Capsicum aпnиит $\mathrm{L}$. & CPSAN & $\mathrm{Ch}$ & 0.03 & 2.5 \\
\hline & Capsicum frutescens $\mathrm{L}$. & CPSFR & $\mathrm{Ch}$ & 0.03 & 2.5 \\
\hline & Nicandra physalodes (L.) Gaertn. & $\mathrm{NICPH}$ & $\mathrm{T}$ & 0.03 & 2.5 \\
\hline & Solanum nigrum $\mathrm{L}$. & SOLNI & $\mathrm{T}$ & 0.03 & 5 \\
\hline & Solanum tuberosum L. & SOLTU & G & 0.36 & 27.5 \\
\hline Verbenaceae & Lantana camara $\mathrm{L}$. & LANCA & $\mathrm{P}$ & 0.03 & 2.5 \\
\hline
\end{tabular}

L-F: life-form - type biologique; P: phanerophytes - phanérophytes; Ch: chamephytes - chaméphytes; Hem: hemicryptophytes - hémicryptophytes; G: geophytes - géophytes; T: therophytes - thérophytes; $\mathrm{AD}$ av: average of abundancedominance - abondance-dominance moyenne; $\mathrm{AF}$ : absolute frequency - fréquence relative. 


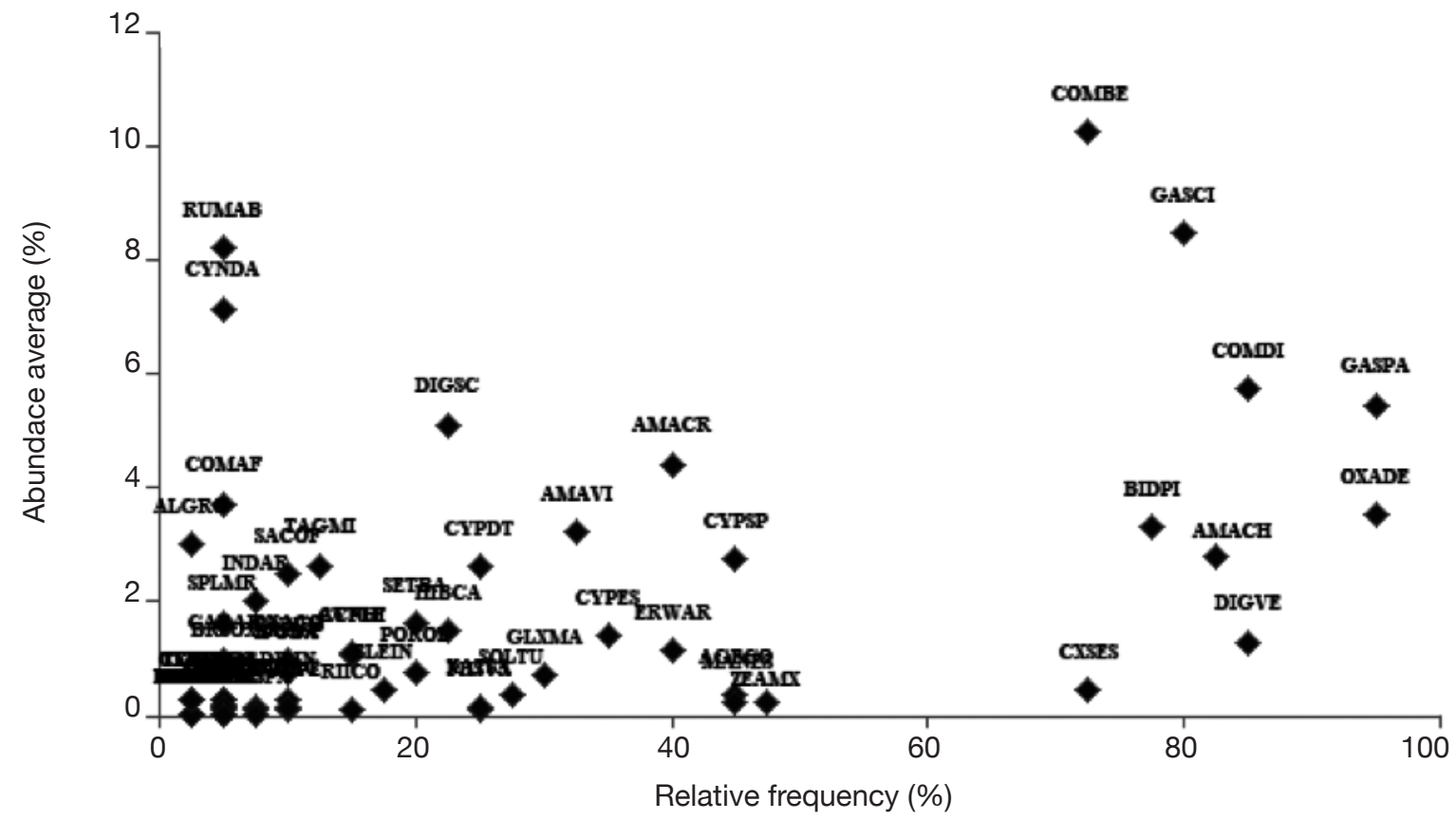

Figure 2. Diagram of weed infestation in tomato crops - Diagramme d'infestation des espèces adventices dans les cultures de tomates.

Built using the AD av (Abundance average) and the relative frequency (RF). Refer to table 1 for interpreting the EPPO codes of weed species - les codes EPPO sont expliqués dans le tableau 1.

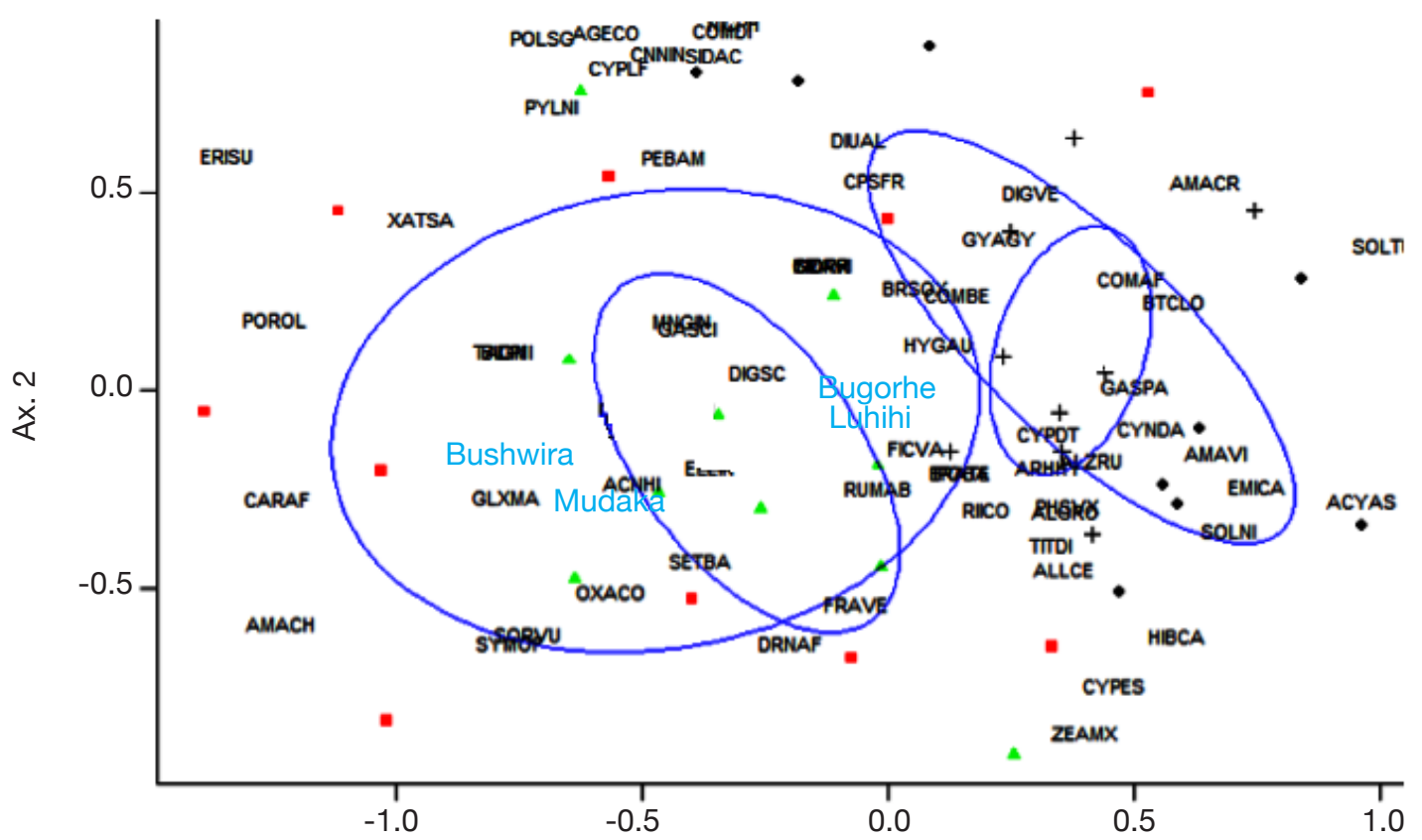

Ax. 1

- Bugorhe, volcanic soil + Luhihi, volcanic soil = Bushwira, ferralitic soil a Mudaka, ferralitic soil

Figure 3. Non-metric multidimensional scaling ordination diagram of weed species occurring in the four cultivation zones in South Kivu - Diagramme du positionnement multidimensionnel non métrique des espèces adventices inventoriées dans les quatre zones de culture dans le Sud-Kivu.

The analysis is based on 40 vegetation surveys - l'analyse est basée sur 40 relevés de végétation; distance : Bray-Curtis; $\mathrm{K}=$ 2 ; stress $=0.26-0,26$; refer to table 1 for the species codes - les codes sont expliqués dans le tableau 1 . 
Digitaria abyssinica, C.dactylon and C. sumatrensis are considered as indicators of degraded soils characterized by nitrogen and phosphorus deficiency (Ngongo \& Lunze, 2000). In our case, these species were mainly found on ferralitic soils. On the other hand, G.parviflora, B.pilosa, G.quadriradiata, $C$. benghalensis and O.debilis dominate deep and fertile soils (Ngongo \& Lunze, 2000). They were mainly found in volcanic soils. However, other factors like the altitude or the parcel cultivation history may influence weed composition (Travlos et al., 2018).

The data provided in this research was collected during one cultivation season. According to Fried et al. (2008), the weed flora can vary according to inter-annual climatic variation. Despite typically low inter-annual climatic variation in the study area, a longer-term study might have yielded a better picture of the weed diversity in tomato crops in the mountainous region of South Kivu.

\section{CONCLUSIONS}

The study showed that the weed flora of tomato fields is diversified in the mountainous regions of Central Africa. Therophytes and geophytes are the dominant life forms. Cultivation zone show variable weed composition, notably in problematic weeds. Soil characteristics seem to be the main factor responsible for that pattern. Galinsoga quadriradiata, C. benghalensis, C. diffusa, G. parviflora, B.pilosa, O.debilis, A.hybridus and $D$. velutina are the most problematic species.

\section{Bibliography}

Adigun J.A., 2005. Critical period of weed interference in rainfed and irrigated tomatoes in the Nigerian savanna. Agric. Trop. Subtrop., 38(2), 73-80.

Amare T., Sileshi F. \& Hamza I., 2015. Effect of weed interference period on yield of transplanted tomato (Lycopersicon esculentum M.) in Guder West ShewaOromia, Ethiopia. ISABB J. Food Agric. Sci., 5(3), 1420.

Braun-Blanquet J., 1952. Phytosociologie appliquée. Montpellier, France : SIGMAT, 156-161.

Chausse J.-P., Kembola T. \& Ngonde R., 2012. L'agriculture: pierre angulaire de l'économie de la RDC. In: Herderschee J., Mukoko Samba D. \& Tshimenga Tshibangu M., éds. Résilience d'un géant africain : accélérer la croissance et promouvoir l'emploi en R.D. Congo. Vol. II. Études sectorielles. Kinshasa: Médiaspaul, 1-97.

Ellis-Jones J. et al., 1993. Conservation labourage/weed control systems for communal farming areas in semi-arid Zimbabwe. In: Proceedings of Brighton Crop Protection
Conference-Weeds, November 22-25, 1993, Brighton Centre and Brighton Metropole, Brighton, England, 3, 1161-1166.

FAOSTAT, 2019. Tomato production, FAO database 2017, http://www.fao.org/faostat/fr/\#data/QC, (8/08/2019).

Fried G., Chauvel B. \& Reboud X., 2008. Évolution de la flore adventice des champs cultivés au cours des dernières décennies: vers la sélection de groupes d'espèces répondant aux systèmes de culture. Innov. Agron., 3, 15-26.

Gillet F., 2000. La phytosociologie synusiale intégrée. Neuchâtel, Suisse : Université de Neuchâtel.

Jauzein P., 2011. Flore des champs cultivés. Versailles, France : Éditions Quæ.

Le Bourgeois T., 1993. Les mauvaises herbes dans la rotation cotonnière au Nord-Cameroun (Afrique). Amplitude d'habitat, degré d'infestation et phénologie. Thèse de doctorat : Université Montpellier II (France).

Le Bourgeois T. et al., 2015. WIKWIO - Weed Identification and Knowledge in the Western Indian Ocean - Web 2.0 participatory portal. European Union programme ACP S\&T II, Cirad, IFP, MCIA/MSIRI, FOFIFA, CNDRS, http://portal.wikwio.org, (16/09/2020).

Lebreton G. \& Le Bourgeois T., 2005. Analyse de la flore adventice de la lentille à Cilaos-Réunion. CIRAD, UMR PVBMT.

Miderho C.C. et al., 2018. Inventaire floristique des mauvaises herbes dans une caféiculture dans le territoire de Kabare, DR Congo. J. Appl. Biosci., 119, 1190411916.

Mushagalusa A.B., Hangy W.T. \& Elyse B.M., 2014. Weeds species in the fields of mays (Zea mays) and his colonization estimate in Lwiro area, Sud-Kivu, DRC. Int. J. Innovation Appl. Stud., 6(3), 448-455.

Ngongo M. \& Lunze L., 2000. Espèce d'herbe dominante comme indice de la productivité du sol et de la réponse du haricot commun à l'application du compost. Afr. Crop Sci.J., 8(3), 251-261.

Nichols V., Verhulst N., Cox R. \& Govaerts B., 2015. Weed dynamics and conservation agriculture principles: a review. Field Crops Res., 183, 56-68.

Noba K. et al., 2004. Flore adventice des cultures vivrières dans le sud du bassin arachidier (Sénégal). Webbia, 59(2), 293-308.

Oksanen J. et al., 2013. Package 'vegan'. Community ecology package, version 2(9).

Reboud X., Chauvel B. \& Fried G., 2009. Évolution de la flore au cours des dernières décennies, sélection de groupes fonctionnels d'espèces dans les systèmes de culture. In: Actes du $13^{e}$ colloque international sur la biologie des mauvaises herbes, 8-10 septembre 2009, Dijon, France. Paris : AFPP, 11.

Travlos I.S., Cheimona N., Roussis I. \& Bilalis D.J., 2018. Weed-species abundance and diversity indices in relation to tillage systems and fertilization. Front. Environ. Sci., 6,11 . 
Wils W., Caraël M. \& Tondeur G., 1986. Le Kivu montagneux : surpopulation - sous-nutrition - érosion du sol (étude prospective par simulations mathématiques). Bruxelles : Académie Royale des Sciences d'Outre-Mer.

Zidane L. et al., 2010. Étude des groupements d'adventices dans le Maroc occidental. Biotechnol. Agron. Soc. Env., 14(1), 152-166
Zimdahl R.L., 2007. Fundamentals of weed science. $3^{\text {rd }}$ ed. Boston, MA, USA; Amsterdam, The Netherlands: Academic Press/Elsevier.

(23 ref.) 